\title{
Polymer cyclization as a general strategy for the emergence of hierarchical nanostructures
}

Chaojian Chen ${ }^{1,2}$, Manjesh Kumar Singh ${ }^{3}$, Katrin Wunderlich ${ }^{1}$, Sean Harvey ${ }^{1}$, Manfred Wagner ${ }^{1}$, George Fytas ${ }^{1,4}$, Kurt Kremer ${ }^{1}$, Debashish Mukherji ${ }^{5}$, David Y. W. Ng${ }^{1 *}$, Tanja Weil ${ }^{1,2 *}$

${ }^{1}$ Max Planck Institute for Polymer Research, Ackermannweg 10, 55128 Mainz, Germany

${ }^{2}$ Ulm University, Albert-Einstein-Allee 11, 89081 Ulm, Germany

${ }^{3}$ Department of Mechanical Engineering, Indian Institute of Technology Kanpur, Kanpur: 208016, Uttar Pradesh, India

${ }^{4}$ Institute of Electronic Structure and Laser, Foundation for Research and Technology, P.O. Box 1527, 71110 Heraklion, Greece

${ }^{5}$ Quantum Matter Institute, University of British Columbia, Vancouver V6T 1Z4, Canada

*Correspondence author. Email: weil@mpip-mainz.mpg.de (T.W.); david.ng@ mpipmainz.mpg.de (D.Y.W.N.)

Abstract: The creation of synthetic polymer nanoobjects with well-defined hierarchical structures is important for a wide range of applications such as nanomaterial synthesis, catalysis, and therapeutics. Inspired by the programmability and precise three-dimensional architectures of biomolecules, we report the strategy of fabricating controlled hierarchical structures through selfassembly of folded synthetic polymers. Linear poly(2-hydroxyethyl methacrylate) of different lengths are folded into cyclic polymers and their self-assembly into hierarchical structures is elucidated by various experimental techniques and molecular dynamics simulations. Based on their structural similarity, macrocyclic brush polymers with amphiphilic block side chains are synthesized, which can self-assemble into wormlike structures and higher-ordered networks. Our work points out the vital role of polymer folding in macromolecular self-assembly and establishes a versatile approach for constructing biomimetic hierarchical assemblies.

One Sentence Summary: Hierarchical wormlike structures and higher-ordered networks can be obtained via biomimetic folding and assembly of synthetic polymers. 
Precise three-dimensional architectures of biomacromolecules such as proteins and DNA have stimulated various new developments in macromolecular chemistry (1-4). Intrigued by the programmability and specificity of intramolecular forces that enable folding of a giant molecular chain $(5,6)$, synthetic chemists have taken several approaches to recreate, in part, notable features that contribute to these unique nanostructures. A simple biomimetic model typically involves a polymer chain consisting of chemical functions that recognize their reactive partner further along the chain, such that the main backbone can fold in a predictable way (7-10). These functions funnel the energy landscape of chain dynamics into, ideally, a single conformation that would otherwise be subjected to randomness and kinetically trapped states. This led to the inception of single chain nanoparticles (SCNPs) (11), which focused on how polymers can be programmed to fold in a regular way akin to the chaperones of proteins (6). The confinement characterized by these SCNPs has discovered newfound capabilities in catalysis, biomedicine, synthetic biology and when doped, offer interesting bulk material properties $(12,13)$.

Despite these advancements, the larger perspective of how folding of a polymer chain can 15 program higher ordered assemblies remains rare $(14,15)$. The concept of assembly driven by the regularity of macromolecules is a critical step for building sophisticated architectures that mimic the ability of proteins to form cellular nanostructures and compartments (16-19). Our study focuses on the emergence of structural complexity by folding of polymer chains forming globules capable of controlled assembly into anisotropic worms and networks. Polymers of 2-hydroxyethyl methacrylate (HEMA) are one of the simplest polymer scaffolds possessing a hydrophobic backbone and a hydrophilic side chain that promotes inter-chain interactions through van der Waals interactions and hydrogen bonds. Folding of the polymer is directed by a single copper catalyzed azide-alkyne cycloaddition at the terminal ends (20). The head-to-tail bite causes the polymer chain, in aqueous solvent, to adopt a self-propagating structure consisting of a hydrophobic core surrounded by the hydroxyl groups of HEMA. The physical properties and the complexity of the propagating structures can be subsequently customized by growing a secondary block-copolymer on each HEMA side chain. Depending on the composition of the blockcopolymer, higher ordered assembly morphology between the unfolded and folded form can be programmed. 
To demonstrate that synthetic polymers can be folded into specific forms for constructing higher-ordered structures, polymers of HEMA (PHEMA) were folded into the cyclic topology, which is a well-established approach to fold polymers (21). Three linear PHEMA samples ( $l$ PHEMA $_{\mathrm{n}}-\mathrm{Br}$, where $\mathrm{n}$ represents the number of repeating units) of different lengths were synthesized via atom transfer radical polymerization (ATRP) using propargyl 2-bromoisobutyrate as the initiator (Fig. 1A). Their respective average molecular weights were calculated from the ${ }^{1} \mathrm{H}$ nuclear magnetic resonance (NMR) spectra as $1670 \mathrm{~g} \mathrm{~mol}^{-1}(\mathrm{n}=11), 2120 \mathrm{~g} \mathrm{~mol}^{-1}(\mathrm{n}=15)$, and $3040 \mathrm{~g} \mathrm{~mol}^{-1}(\mathrm{n}=22)$, which fit well to the design (figs. S1-S3, table S1). GPC results show relatively narrow molecular weight distributions of $1.36 \sim 1.39$ (Fig. 1B). The molecular weights determined by GPC are higher, which is due to the different hydrodynamic sizes of poly(methyl methacrylate) (PMMA) standards and PHEMA in DMF (22). Next, the bromine ends of these polymers were transformed to azide groups by reacting with sodium azide, which was confirmed by the appearance of the characteristic peak of azide at $2121 \mathrm{~cm}^{-1}$ in the Fourier-transform infrared (FTIR) spectra of the product (l-PHEMAn- $\left.N_{3}\right)$ (Fig. 1C and fig. S4).
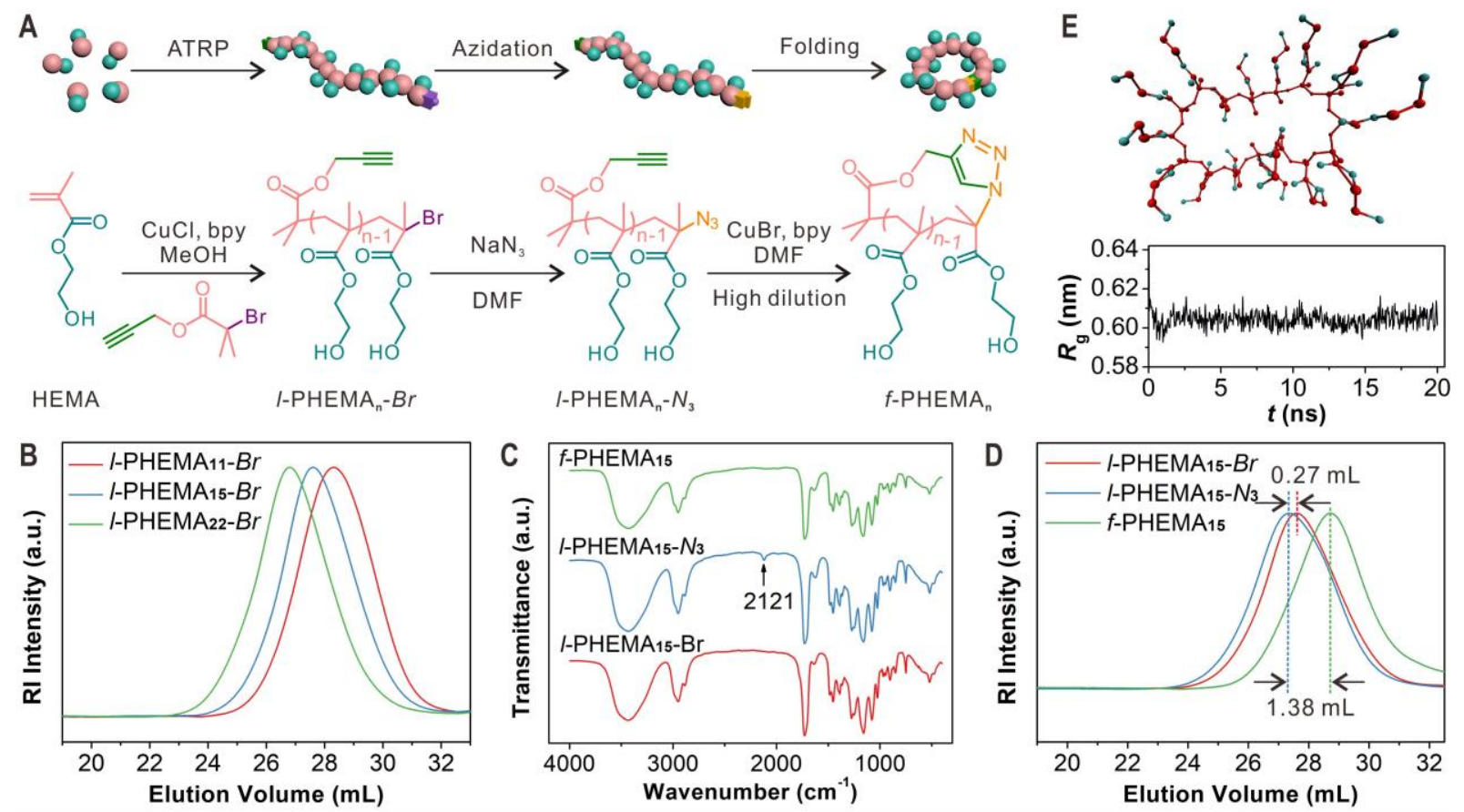

Fig. 1. Synthesis and folding of PHEMA. (A) Scheme of the synthesis of $l$-PHEMA $-B r$ and its azidation and folding into the cyclic topology. (B) GPC curves (eluent: DMF; standard: PMMA) of $l$-PHEMAn- $B r$ with different repeating units. (C) FTIR spectra, and (D) GPC curves of $l$ PHEMA15- $B r, l$-PHEMA15- $N_{3}$, and $f$-PHEMA15. (E) The top and bottom panels show a simulation snapshot and the time evolution of $R_{\mathrm{g}}$ of a single $f$-PHEMA 15 in pure water, respectively. 
Subsequently, the heterobifunctional PHEMA with azide and alkyne ends was folded via Huisgen cycloaddition under high dilution conditions (20). In a typical experiment, $\mathrm{CuBr}$ and 2,2'bipyridyl (bpy) were added into a Schlenk tube loaded with DMF, which was degassed through two freeze-pump-thaw cycles. To avoid intermolecular reactions, the degassed DMF solution of $l$ PHEMA $_{n}-N_{3}$ was added into the catalyst solution at a slow speed of $0.16 \mathrm{~mL} \mathrm{~h}^{-1}$ via a syringe pump. As shown in Fig. 1C, the asymmetric stretching peak of the azide group at $2121 \mathrm{~cm}^{-1}$ vanished after this step. This indicates that the click reaction between azide and alkyne groups was successful, which was also proven by ${ }^{1} \mathrm{H}$ NMR (figs. S5-S7). We also proved the structure for all the products in each step by $2 \mathrm{D}{ }^{1} \mathrm{H},{ }^{1} \mathrm{H}$ correlation spectroscopy and $2 \mathrm{D}{ }^{1} \mathrm{H},{ }^{1} \mathrm{H}$ nuclear Overhauser effect spectroscopy methods (figs. S8-S16).

The obtained polymers were further characterized by GPC (Fig. 1D and fig. S17) and ${ }^{1} \mathrm{H}$ diffusion ordered NMR spectroscopy (figs. S18-S23). Both techniques demonstrate that the hydrodynamic volumes of all three samples decreased after the click reaction, which confirms that linear PHEMA polymers were intramolecularly folded into the cyclic topology. It should be 15 mentioned that no shoulder peak in the higher molecular regions was observed in the GPC curves indicating no linear polymers left or oligomers formed. To investigate if a single $f$-PHEMA 15 retains its well-defined cyclic structure in pure water, we performed an all-atom molecular dynamics simulation (fig. S24). In Fig. 1E we show the snapshot of a $f$-PHEMA 15 and the corresponding radius of gyration $\left(R_{\mathrm{g}}\right)$. It can be appreciated that a ring structure remains rather stable in water.

Linear PHEMA is generally regarded as a water-swellable polymer (23). Using a dialysis method (24), we compared the self-assembly behaviors of linear and folded PHEMA. Briefly, the polymer was dissolved in methanol and then deionized water was added dropwise. The mixture solution was loaded into a dialysis membrane $\left(\mathrm{MWCO}=1000 \mathrm{~g} \mathrm{~mol}^{-1}\right)$ and dialyzed against water. 25 As shown in Fig. 2A and fig. S25, solutions of the linear polymers turned turbid after one hour and some gel-like precipitation was observed three days later. Surprisingly, solutions of the folded polymers remain clear, a first indication that they can stabilize themselves in pure water. 


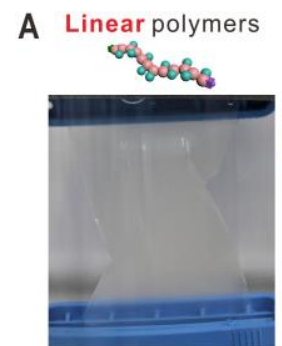

I-PHEMA $15-\mathrm{Br}$

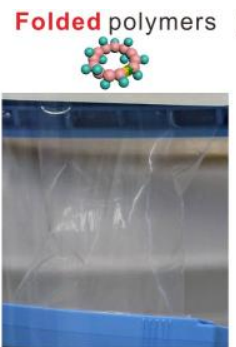

f-PHEMA

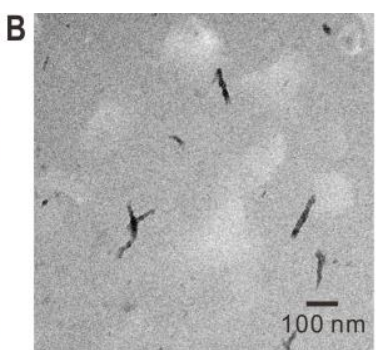

$f$-PHEMA

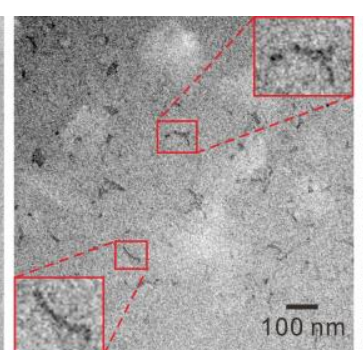

$f-\mathrm{PHEMA}_{15}$

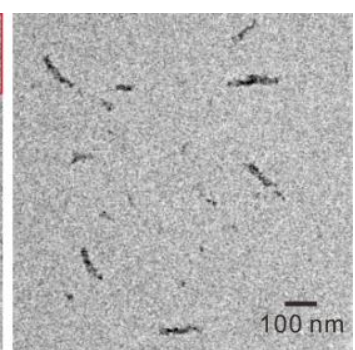

$f$-PHEMA

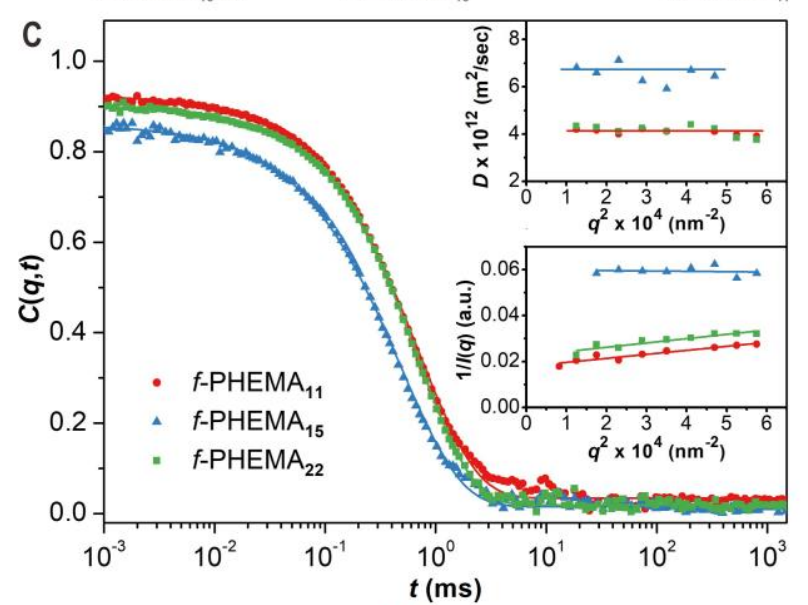

E

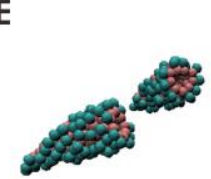

$\mathrm{F}$

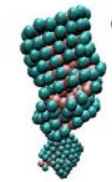

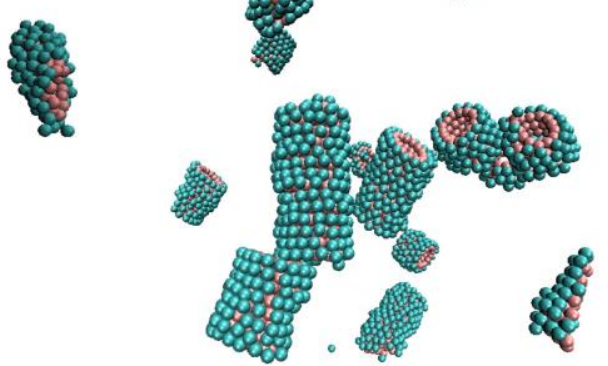

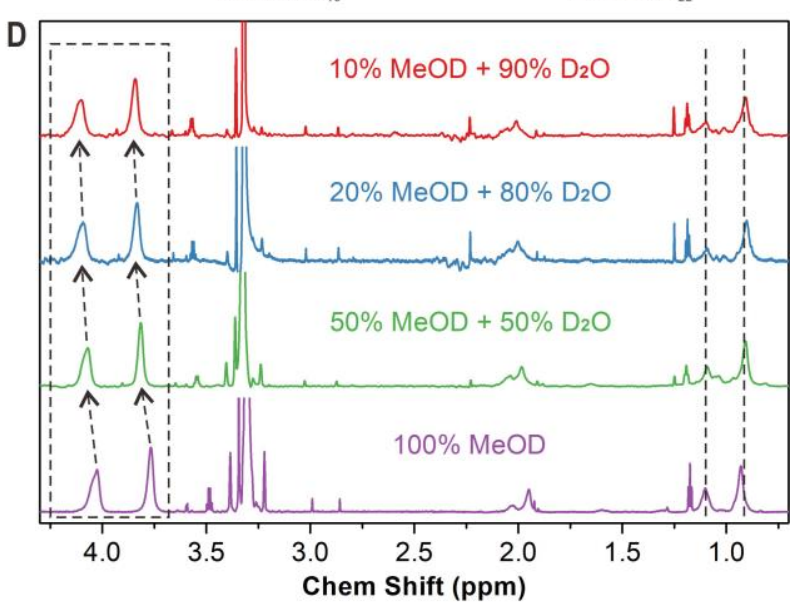

G

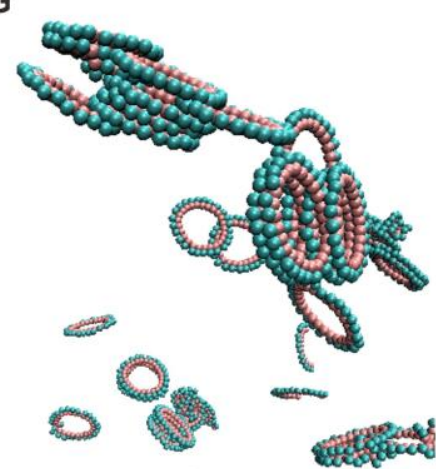

Fig. 2. Self-assembly of linear and folded polymers. (A) Solutions of $l$-PHEMA $15-B r$ and $f$ PHEMA $_{15}$ after dialysis against deionized water for one hour. (B) TEM images showing the assemblies of $f$-PHEMA $11, f$-PHEMA 15 , and $f$-PHEMA 22 . (C) Relaxation functions $C(q, t)$ for the translation motion of $f$-PHEMA $11, f$-PHEMA 15 , and $f$-PHEMA 22 in water at $1 \mathrm{mg} \mathrm{mL}^{-1}$ and $293 \mathrm{~K}$ at a scattering angle of $90^{\circ}$ corresponding to scattering wave vector $q=0.0187 \mathrm{~nm}^{-1}$. Upper inset: The translation diffusion coefficient, $D$ as a function of $q^{2}$ with the solid line indicating a virtually $q$ independent $D$. Lower inset: $1 / I(q)$ as a function of $q^{2}$. (D) ${ }^{1} \mathrm{H}$ NMR spectra $(850 \mathrm{MHz}, 298.3 \mathrm{~K})$ of $f$-PHEMA 15 in mixtures of MeOD and $\mathrm{D}_{2} \mathrm{O}$ with gradually tuned volume ratios. The chemical shifts were calibrated using tetramethylsilane as an internal standard. (E-G) Molecular dynamic simulation snapshots showing the self-assembly of a model simulation replica of $f$-PHEMAn with gradually increased ring sizes, i.e., $n=9(E), n=15(F)$, and $n=25(G)$.

Transmission electron microscopy (TEM) images reveal the formation of wormlike structures from the folded polymers (Fig. 2B and figs. S26-S28). For linear polymers of the same 
molecular weights, only irregular aggregates were observed showing the significant role of folding on the self-assembly (figs. S26-S28). We further confirmed the generation of stable assemblies from folded PHEMA by dynamic light scattering (DLS). Interestingly, both TEM and DLS results suggest $f$-PHEMA 15 with a middle ring size formed the smallest assemblies. The hydrodynamic radius $R_{\mathrm{h}}$ for assemblies of $f$-PHEMA $11, f$-PHEMA 15 , and $f$-PHEMA 22 were determined as $54 \pm 2$ nm, $36 \pm 2 \mathrm{~nm}$, and $58 \pm 3 \mathrm{~nm}$, respectively (Fig. 2C and figs. S29-S31). The large size of the $f$ -

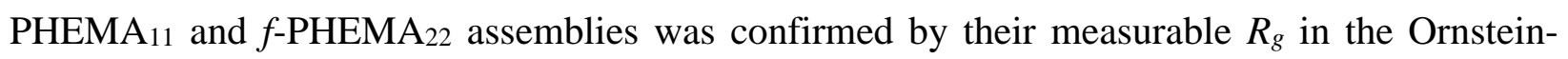
Zernike, $I(q)^{-1}$ vs $q^{2}$ (lower inset to Fig. $2 \mathrm{C}$ ): $I(q)$ of the smaller $f$-PHEMA15 case is virtually $q$ independent (25). The calculated $R_{g}=50 \mathrm{~nm}(59 \mathrm{~nm})$ for the $f$-PHEMA11 $(f$-PHEMA22) assemblies are very similar to the values of $R_{\mathrm{h}}$ suggesting compact structures much larger than the single rings (Fig. 1E). For linear PHEMA, DLS experiment was not possible due to the presence of large aggregates.

The unique assembly behaviors of cyclic PHEMA can be ascribed to the rearrangement of the atomic distribution by polymer folding. Nile Red loading experiments indicated the formation 15 of hydrophobic microenvironments during the assembly (fig. S32). The measured critical aggregation concentrations of $f$-PHEMA $\left(\sim 0.01 \mathrm{mg} \mathrm{mL}^{-1}\right)$ are lower than that of many amphiphilic polymers $\left(0.02 \sim 0.2 \mathrm{mg} \mathrm{mL}^{-1}\right)(24,26)$, showing the strong assembly trend of folded polymers. Furthermore, we performed NMR measurements for $f$-PHEMA $\mathrm{n}$ in solvent mixtures of methanol$\mathrm{d}_{4}(\mathrm{MeOD})$ and deuterium oxide $\left(\mathrm{D}_{2} \mathrm{O}\right)$ with gradually tuned compositions (Fig. 2D and fig. S33). When the amount of $\mathrm{D}_{2} \mathrm{O}$ increased, the signals of the methyl groups in the backbone did not change. However, the peaks from the methylene groups (3.77 and $4.03 \mathrm{ppm}$ ) in hydroxyethyl side chains shifted. This result indicates that the backbones of $f$-PHEMAn were packed in the core and the side chains on the surface enabling them to freely interact with the polar solvent.

To further elucidate the self-assembly, we performed the molecular dynamics simulations 25 of an implicit solvent generic model. In this model, the quantities are expressed in the units of energy $\varepsilon$, length $\sigma$ and time $\tau$. The choice of the generic model parameter of a ring polymer is inspired by the structural stability and the solubilities of the individual residues in pure water, as observed for a $f$-PHEMA 15 (Fig. 1E) $(27,28)$. For this purpose, we investigated three ring sizes (n $=9,15$ and 25) at a constant mass density. The simulation snapshots for $\mathrm{n}=9$ and 15 show 30 wormlike micellar structures (Figs. 2E and F). Here, a self-assembled structure is dictated by the 
competition between the energy penalty of forming an aggregate of a particular size from the homogeneous mixture and the surface energy (29). In our case, while we abstain from discussing the details of aggregation kinetics, we note that the sizes of the largest aggregates stabilize after a time $t \sim 10^{4} \tau$ in all cases. We find that the length $(L)$, diameter $(d)$, and $R_{\mathrm{g}}$ of the largest aggregates are $L \sim 33.5 \sigma, d \sim 3.3 \sigma$ and $R_{\mathrm{g}} \sim 9.9 \sigma$ for $\mathrm{n}=9$, and $L \sim 14.2 \sigma, \mathrm{d} \sim 4.1 \sigma$ and $R_{\mathrm{g}} \sim 5.0 \sigma$ for $\mathrm{n}=15$. Furthermore, from $L$ and $d$ values of $\mathrm{n}=9$ and the analytical expression (30), we have estimated $R_{\mathrm{h}} \sim 10.7 \sigma$. These are consistent with the experimental trends, i.e., (a) $R_{\mathrm{g}} \sim R_{\mathrm{h}}$ and (b) $R_{\mathrm{g}}$ decreases with increasing n (Fig. 2C). For $n=25$ a sandwich-like stacking is observed (Fig. 2G), which is not surprising given that the hydrophobic part of this ring is rather large and thus multiple chains can stack in a side-wise sandwich structure. The data in Fig. 2G may further suggest that there exists an upper bound of $\mathrm{n}$ that can facilitate a well-defined stable self-assembly in these systems.

The above results clearly show that even homopolymers with a very simple structure can assemble into higher-ordered nanostructures after molecular folding. Therefore, we believe, like polypeptides and nucleic acids, common synthetic polymers can also be programmed into controlled nanoobjects with multiple levels of defined architectures through folding and assembly. As a proof-of-concept, we designed macrocyclic brush polymers with amphiphilic polystyreneblock-poly(acrylic acid) (PS-b-PAA) side chains. PS and PAA were selected because they are broadly representative of hydrophobic and hydrophilic polymers, and PS- $b$-PAA is one of the most intensively studied systems in the field of macromolecular self-assembly (26).

The cyclic macroinitiator $f$-P(HEMA-Br) 22 was synthesized by attaching ATRP initiators to hydroxyl groups of $f$-PHEMA22 (Fig. 3A). Hydrophobic PS and poly(tert-butyl acrylate) (PtBA) were consecutively grafted from the cyclic macroinitiator. The P $t$ BA block was then hydrolyzed by trifluoroacetic acid into PAA, generating cyclic brush polymers with amphiphilic side chains $[f \text {-P(HEMA- } g \text {-PS }-b \text {-PAA })_{22}$, where $\mathrm{x}$ and y represent the numbers of repeating units for PS and PAA, respectively]. These cyclic brush polymers can be regarded as the simplest folded form of the corresponding linear brush polymers. As shown in Fig. 3B and C, we synthesized four cyclic brush polymers (CB-1 to CB-4). For comparison, a block copolymer (BC) and a linear brush polymer (LB) with comparable compositions were also prepared. All products in each step have been systematically characterized by NMR and FTIR spectroscopies as well as GPC (figs. S34- 
S48, tables S2-S4). Analysis of cyclic brush polymers by GPC shows narrow size distributions with $Ð$ in the range of 1.17 to 1.48 , indicating that they can be used as uniform building blocks.
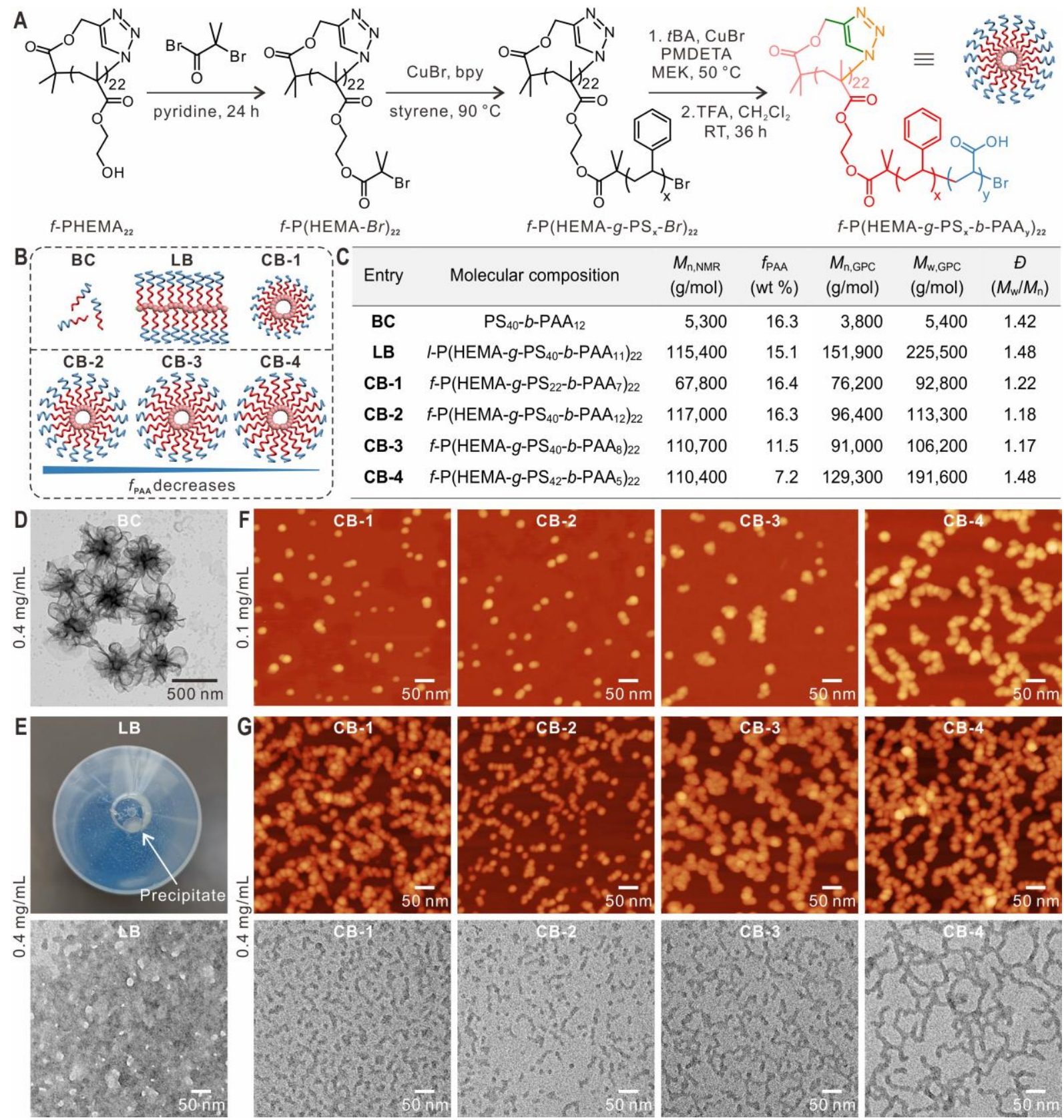

Fig. 3. Hierarchical self-assembly of cyclic brush polymers. (A) Scheme for the synthesis of cyclic brush polymers $f$-P(HEMA- $g$-PS $-b$-PAAy $)_{n}$. (B) Schematic illustration and (C) molecular parameters of block copolymer BC, linear brush polymer $\mathbf{L B}$, and four cyclic brush polymers (CB1 to CB-4). (D) TEM image showing the self-assembly of BC at $0.4 \mathrm{mg} \mathrm{mL}^{-1}$ in water. (E) Optical (top) and TEM (bottom) images showing the aggregation of $\mathbf{L B}$ in water. (F) AFM images showing the self-assembly of CB-1 to CB-4 at $0.1 \mathrm{mg} \mathrm{mL}^{-1}$ in water. (G) AFM (top) and TEM (bottom) images showing the self-assembly of cyclic brush polymers into 1D wormlike structures and hierarchical networks at $0.4 \mathrm{mg} \mathrm{mL}^{-1}$ in water. 
Using a similar dialysis method, we investigated the self-assembly behavior of these amphiphilic cyclic brush polymers (CB-1 to CB-4) in water and compared them with BC and LB. The polymers were first dissolved in DMF, which is a good solvent for both PS and PAA. After adding the same amount of deionized water dropwise, the mixture solutions were dialyzed against water for three days and then tuned to desired concentrations. As shown in Fig. 3D and fig. S49, block copolymer BC self-assembled into lamellae, vesicles, and large compound vesicles at different concentrations due to its high weight fraction of hydrophobic PS. In comparison, the LB counterpart to the polymerized form of $\mathbf{B C}$ could not form ordered structures and precipitation was observed at $0.4 \mathrm{mg} \mathrm{mL}^{-1}$ (Fig. 3E, figs. S50-S51).

Interestingly, cyclic brush polymers with similar compositions but different topologies demonstrated drastically different self-assembly behaviors. We found that the polymer concentration and the weight fraction of PAA $\left(f_{\mathrm{PAA}}\right)$ are two important factors on the assembly. Discrete structures were observed by atomic force microscopy (AFM) and TEM for all samples when the concentrations were $0.1 \mathrm{mg} \mathrm{mL}^{-1}$ (Fig. 3F). The smallest particles in the AFM images show the same height of about $5 \mathrm{~nm}$ (figs. S52-S54), which corresponds with the size of single cyclic brushes. Other bigger nanoobjects can be ascribed to dimers, trimers, or oligomers of cyclic brush polymers. Importantly, the boundary between cyclic brush polymers can be visualized by AFM, which clearly shows the assembly of cyclic brush polymers in a layer-by-layer manner (Fig. 3F and figs. S52-S55). When the concentrations increased to $0.4 \mathrm{mg} \mathrm{mL}^{-1}$, wormlike structures and hierarchical networks were obtained (Fig. 3G and figs. S56-S59). Unlike simple diblock copolymers that form wormlike micelles only in a narrow window of hydrophobic-hydrophilic ratios, folded polymers with a broad range of compositions can assemble into wormlike structures. More importantly, these wormlike structures are generated via stepwise folding and modular 25 assembly, allowing the modulation of the internal structure and overall dimension in each step. For cyclic brush polymers with similar side chain lengths (CB-2 to CB-4), the assemblies became longer with the decrease of $f_{\text {PAA. }}$ By customizing the block constituents, proportion and length, the architectural outcome can be tuned.

To investigate these interesting structures, we also performed a set of generic simulations of a replica of the cyclic brush polymers for five different concentrations $(c)$. The data for two concentrations is shown in Fig. 4A-B and the complete data is shown in fig. S60 and movies S1- 
S10. Here, the side chains greatly hinder the self-assembly. For $c=0.0002 \sigma^{-3}$, discrete oligomers are observed attaining a maximum size of $\sim 8.5 \sigma$ (Fig. 4A) that is smaller than the model $f$ PHEMA9 (Fig. 2E). Moreover, for $c=0.0009 \sigma^{-3}$, the side chains can interdigitate and thus start to form a network-like assembly (Fig. 4B). These observations are consistent with the trends observed in the AFM and SEM measurements.

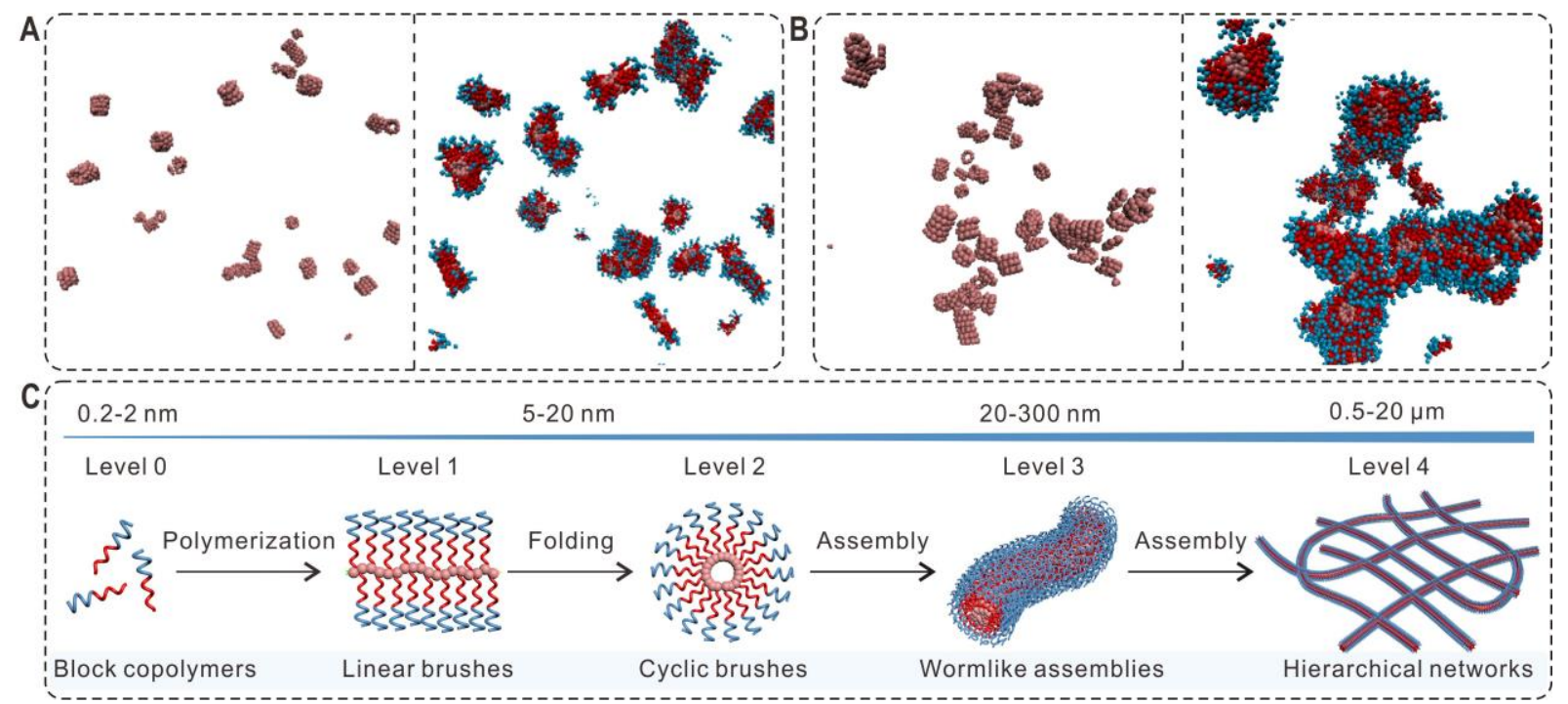

Fig. 4. Emergence of structural complexity by self-assembly of cyclic brush polymers. (A-B) Molecular simulation results showing the self-assembly of cyclic brush polymers for the two different concentrations, $c=0.0002 \sigma^{-3}(\mathrm{~A})$ and $0.0009 \sigma^{-3}(\mathrm{~B})$. The left panels show only the 10 hydrophobic backbones and the right panels illustrate the full molecules. (C) Formation of hierarchical structures by synthetic polymers via biomimetic folding and self-assembly.

We have therefore established a biomimetic strategy for the construction of hierarchical nanostructures via self-assembly of folded polymers. By rearranging atomic distribution of polymers to form specific preliminary structures, this modular approach is particularly powerful for preparing wormlike assemblies from synthetic polymers including common homopolymers and block copolymers. Various parameters including the polymer composition, molecular weight and primary structure can be used to manipulate the overall structure of the assemblies. The selfassembly pathway for the hierarchical networks is a versatile and stage-distinct platform to mimic the increasing complexity in the folding of polypeptides into 3D precise protein structures (Fig. 4C). The combination of polymer folding and self-assembly can therefore serve as a novel avenue for fabricating the next-generation hierarchical structures of unprecedentedly complex shapes which cannot be achieved by traditional methods. 


\section{References and Notes:}

1. M. Heim, L. Romer, T. Scheibel, Hierarchical structures made of proteins. The complex architecture of spider webs and their constituent silk proteins. Chem. Soc. Rev. 39, 156-164 (2010).

2. N. C. Seeman, H. F. Sleiman, DNA nanotechnology. Nat. Rev. Mater. 3, 17068 (2018).

3. C. J. Chen, D. Y. W. Ng, T. Weil, Polymer bioconjugates: Modern design concepts toward precision hybrid materials. Prog. Polym. Sci. 105, 101241 (2020).

4. C. J. Chen, K. Wunderlich, D. Mukherji, K. Koynov, A. J. Heck, M. Raabe, M. Barz, G. Fytas, K. Kremer, D. Y. W. Ng, T. Weil, Precision Anisotropic Brush Polymers by Sequence Controlled Chemistry. J. Am. Chem. Soc. 142, 1332-1340 (2020).

5. P. W. K. Rothemund, Folding DNA to create nanoscale shapes and patterns. Nature 440, 297302 (2006).

6. D. Balchin, M. Hayer-Hartl, F. U. Hartl, In vivo aspects of protein folding and quality control. Science 353, aac4354 (2016).

7. B. V. K. J. Schmidt, N. Fechler, J. Falkenhagen, J. F. Lutz, Controlled folding of synthetic polymer chains through the formation of positionable covalent bridges. Nat. Chem. 3, 234-238 (2011).

8. N. Hosono, M. A. J. Gillissen, Y. C. Li, S. S. Sheiko, A. R. A. Palmans, E. W. Meijer, Orthogonal Self-Assembly in Folding Block Copolymers. J. Am. Chem. Soc. 135, 501-510 (2013).

9. P. J. M. Stals, Y. C. Li, J. Burdynska, R. Nicolay, A. Nese, A. R. A. Palmans, E. W. Meijer, K. Matyjaszewski, S. S. Sheiko, How Far Can We Push Polymer Architectures? J. Am. Chem. Soc. 135, 11421-11424 (2013).

10. Y. Tezuka, Topological Polymer Chemistry Designing Complex Macromolecular Graph Constructions. Acc. Chem. Res. 50, 2661-2672 (2017).

11. S. Mavila, O. Eivgi, I. Berkovich, N. G. Lemcoff, Intramolecular Cross-Linking Methodologies for the Synthesis of Polymer Nanoparticles. Chem. Rev. 116, 878-961 (2016).

12. Y. L. Liu, T. Pauloehrl, S. I. Presolski, L. Albertazzi, A. R. A. Palmans, E. W. Meijer, Modular Synthetic Platform for the Construction of Functional Single-Chain Polymeric Nanoparticles: From Aqueous Catalysis to Photosensitization. J. Am. Chem. Soc. 137, 13096-13105 (2015).

13. M. Gonzalez-Burgos, A. Latorre-Sanchez, J. A. Pomposo, Advances in single chain technology. Chem. Soc. Rev. 44, 6122-6142 (2015).

14. M. Schappacher, A. Deffieux, Synthesis of macrocyclic copolymer brushes and their selfassembly into supramolecular tubes. Science 319, 1512-1515 (2008).

15. E. B. Trigg, T. W. Gaines, M. Marechal, D. E. Moed, P. Rannou, K. B. Wagener, M. J. Stevens, K. I. Winey, Self-assembled highly ordered acid layers in precisely sulfonated polyethylene produce efficient proton transport. Nat. Mater. 17, 725-731 (2018).

16. A. H. Groschel, A. Walther, T. I. Lobling, F. H. Schacher, H. Schmalz, A. H. E. Muller, Guided hierarchical co-assembly of soft patchy nanoparticles. Nature 503, 247-251 (2013).

17. H. B. Qiu, Y. Gao, C. E. Boott, O. E. C. Gould, R. L. Harniman, M. J. Miles, S. E. D. Webb, M. A. Winnik, I. Manners, Uniform patchy and hollow rectangular platelet micelles from crystallizable polymer blends. Science 352, 697-701 (2016).

18. H. B. Qiu, Z. M. Hudson, M. A. Winnik, I. Manners, Multidimensional hierarchical selfassembly of amphiphilic cylindrical block comicelles. Science 347, 1329-1332 (2015). 
19. M. J. Huang, C. H. Hsu, J. Wang, S. Mei, X. H. Dong, Y. W. Li, M. X. Li, H. Liu, W. Zhang, T. Z. Aida, W. B. Zhang, K. Yue, S. Z. D. Cheng, Selective assemblies of giant tetrahedra via precisely controlled positional interactions. Science 348, 424-428 (2015).

20. B. A. Laurent, S. M. Grayson, An efficient route to well-defined macrocyclic polymers via "Click" cyclization. J. Am. Chem. Soc. 128, 4238-4239 (2006).

21. F. M. Haque, S. M. Grayson, The synthesis, properties and potential applications of cyclic polymers. Nat. Chem. 12, 433-444 (2020).

22. J. V. M. Weaver, I. Bannister, K. L. Robinson, X. Bories-Azeau, S. P. Armes, M. Smallridge, P. McKenna, Stimulus-responsive water-soluble polymers based on 2-hydroxyethyl methacrylate. Macromolecules 37, 2395-2403 (2004).

23. K. L. Robinson, M. A. Khan, M. V. D. Banez, X. S. Wang, S. P. Armes, Controlled polymerization of 2-hydroxyethyl methacrylate by ATRP at ambient temperature. Macromolecules 34, 3155-3158 (2001).

24. C. J. Chen, G. Y. Liu, X. S. Liu, S. P. Pang, C. S. Zhu, L. P. Lv, J. Ji, Photo-responsive, biocompatible polymeric micelles self-assembled from hyperbranched polyphosphate-based polymers. Polym. Chem. 2, 1389-1397 (2011).

25. K. Wunderlich, A. Larsen, J. Marakis, G. Fytas, M. Klapper, K. Mullen, Controlled Hydrogel Fiber Formation: The Unique Case of Hexaphenylbenzene-Poly(ethylene glycol) Amphiphiles. Small 10, 1914-1919 (2014).

26. Y. Y. Mai, A. Eisenberg, Self-assembly of block copolymers. Chem. Soc. Rev. 41, 5969-5985 (2012).

27. C. C. De Silva, P. Leophairatana, T. Ohkuma, J. T. Koberstein, K. Kremer, D. Mukherji, Sequence transferable coarse-grained model of amphiphilic copolymers. J. Chem. Phys. 147, (2017).

28. Y. N. Zhao, M. K. Singh, K. Kremer, R. Cortes-Huerto, D. Mukherji, Why Do Elastin-Like Polypeptides Possibly Have Different Solvation Behaviors in Water-Ethanol and Water-Urea Mixtures? Macromolecules 53, 2101-2110 (2020).

29. D. Mukherji, C. F. Abrams, Microvoid formation and strain hardening in highly cross-linked polymer networks. Phys. Rev. E 78, 050801 (2008).

30. W. Vandesande, A. Persoons, The Size and Shape of Macromolecular Structures Determination of the Radius, the Length, and the Persistence Length of Rodlike Micelles of Dodecyldimethylammonium Chloride and Bromide. J. Phys. Chem. 89, 404-406 (1985).

\section{Acknowledgments:}

M.K.S. thanks generous allocation of computational time at the IIT Kanpur computer facility where most generic simulations were performed. D.M. thanks the ARC Sockeye supercomputing facility where the all atom simulations were performed. Funding: The authors acknowledge financial support from Deutsche Forschungsgemeinschaft (DFG, German Research Foundation) Project number 213555243 - SFB 1066 (A6). C.C. is grateful for a doctoral fellowship from Promotionskolleg Pharmaceutical Biotechnology of Ulm University funded by the state of BadenWürttemberg. D.M. thanks the Canada First Research Excellence Fund (CFREF) for the financial support. Author contributions: T.W. acquired funding for the project. T.W. and D.Y.W.N. supervised the project and corrected the manuscript. C.C. initiated the idea, performed the experiments, and wrote the manuscript. M.K.S., K.K. and D.M. performed the molecular simulations. K.W. and G.F. interpreted the light scattering results. S.H. performed the AFM. M.W. 
provided the NMR expertise. Competing interests: The authors declare no competing interests. Data and materials availability: All data is available in the main text or the supplementary materials.

\section{Supplementary Materials:}

$5 \quad$ Materials and Methods

Supplementary Text

Figures $\mathrm{S} 1$ to $\mathrm{S} 60$

Tables S1 to S4

Movies S1 to S10

10

References (31-42) 